\title{
Image Analysis of DNA Repair and Apoptosis in Tumor Cells with Differing Sensitivity to DNA Damage
}

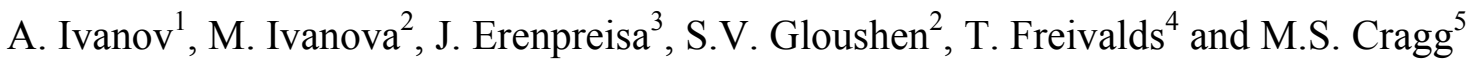 \\ ${ }^{1}$ Paterson Institute of Cancer Research, Manchester, UK; \\ ${ }^{2}$ Belarussian State University, Minsk, Belarussia; \\ ${ }^{3}$ Latvian Biomedicine Centre, Riga, Latvia; \\ ${ }^{4}$ Latvian Institute of Experimental and Clinical Medicine, Riga, Latvia; \\ ${ }^{5}$ Tenovus Research Laboratory, Southampton University School of Medicine, Southampton, UK
}

\begin{abstract}
Homologous recombination of DNA double strand breaks was previously found to be protective against apoptosis in tumor cells lacking p53 function. Here, we studied the spatial and temporal relationship between the two processes in a pair of lymphoblastoid cell lines with wild-type or mutant p53 status. Clonogenic assays revealed that p53 mutant WI-L2-NS cells were $\sim$ ten-fold more resistant to $X$-ray damage than p53 wild type TK6 cells and displayed 2-3 times lower levels of apoptosis $24 \mathrm{~h}$ after irradiation. The kinetics of DNA damage and repair after irradiation ( $5 \mathrm{~Gy}$ ) were assessed by immunofluorescent staining for g-H2AX and Rad51, and DNA stained with DAPI. Using image analysis in the three (red/green/blue) fluorescence channels we found that repair foci were more prevalent in the p53 mutant radioresistant WI-L2-NS cells at many time points: 2.2 versus 1 per nucleus at $5 \mathrm{~min}, 3.2$ versus 1.6 at $6 \mathrm{~h}$, and 6.1 versus 3.6 at $72 \mathrm{~h}$ post-irradiation although the number of repair foci were equal at $24 \mathrm{~h}(\mathbf{8 . 8 )}$. Furthermore, the average size of foci in TK6 cells was smaller at all times post-irradiation $(p<0.001)$. In contrast to the functional repair foci which were characterised by the co-localisation and high concentration of $\mathrm{g}$ H2AX and Rad51, in pre-apoptotic cell nuclei, foci with greater quantities of $\mathrm{g}-\mathrm{H} 2 \mathrm{AX}$ relative to $\mathrm{Rad51}$ were prevalent. The $\mathrm{g}$ H2AX-predominant foci colocalized with little or no Rad51 and fused in a pattern typical of apoptotic chromatin. This pattern was seen more often in TK6 cells, where at $6 \mathrm{~h}$ the sum area occupied by g-H2AX was seven-fold higher than that of the Rad51 label. In contrast, WI-L2-NS cells displayed approximately equal areas of both components. These data suggest that formation of stable functional repair foci topologically protects the chromatin from relaxation and initiation of apoptotic fragmentation.
\end{abstract}

Keywords - tumors, DNA repair, apoptosis, image analysis.

\section{INTRODUCTION}

Tumor cells lacking p53 function are selected during tumor progression and are resistant to therapy with DNA damaging agents. Homologous recombination (HR) of DNA double strand breaks (DSB) was previously found to be abnormally enhanced in p53 mutated tumor cells resulting in a reduction in tumor cell death through apoptosis $[1,2]$. Although it is generally accepted that failed DNA repair leads to apoptosis, the spatial relationship between the two processes and its dependence on p53 status is unknown. In mammalian cells, the signaling cascade downstream of DNA damage phosphorylates histone H2AX (termed g$\mathrm{H} 2 \mathrm{AX}$ ) at sites of DSB. In turn, g-H2AX plays a critical role in the organization of DNA repair foci by helping to recruit DNA repair factors involved in HR, such as the recombinase Rad51 [3]. g-H2AX forms at sites on the chromatin where DSB are present, including those sites where extensive apoptotic DNA fragmentation is occuring. Therefore, we applied immunofluorescent staining of g-H2AX and Rad51 and used image analysis to study the topological and kinetic relationships between HR and apoptosis, in a pair of linked human lymphoblastoid cell lines with wild-type (wt) or mutant (mut) p53 status.

\section{MATERIALS AND METHODS}

Human lymphoblatoid cells lines TK6 and WI-L2-NS were obtained from the ATCC and grown in suspension in RPMI medium with $10 \%$ fetal calf serum. WI-L2-NS is a p53 mutated cell-line derived from the same source as the p53 wt TK6. Cells were irradiated using a Gulmay D3 225 $\mathrm{X}$-ray source with a dose rate of $0.77 \mathrm{~Gy} /$ minute. Samples were analysed by flow cytometry and immunofluorescent staining for $\mathrm{g}-\mathrm{H} 2 \mathrm{AX} / \mathrm{Rad} 51 / \mathrm{DNA}$ as detailed previously (Ivanov et al., 2003). Images were obtained by Axiocam (1300x1030) equipped with a calibrated Sony DXC 390P colour video camera. Image analysis was performed using Image Pro Plus 4.1 software (Media Cybernetics; REO 2001, Riga, Latvia). To discriminate DNA repair foci, the image was segmentated using 180-255 empirically chosen intensity levels. Students t- and non-parametric sign-test were used for statistical analysis of measurement results. 


\section{Results}

Clonogenic assay: Clonogenic assays from three independent experiments showed that the p53 mut WI-L2-NS cells are approximately ten-fold more resistant to X-ray radiation than the related $\mathrm{p} 53 \mathrm{wt}$ TK6 cells.

Kinetics of the cell cycle: DNA flow cytometry revealed that $60 \%$ of irradiated TK6 cells and about $75 \%$ of WI-L2-NS cells have passed the first S-phase and arrived in the G2 arrest compartment at $24 \mathrm{~h}$ post 5 Gy irradiation (in control, G2fraction comprised $22-26 \%$, in the both tumours). Mitotic cycling restarted then, albeit at a low level, and returned to the normal level between day 5 to 7 . Between d.2 to d.5, WIL2-NS formed about $20 \%$ of polyploid cells $(>4 \mathrm{C})$, while TK6 produced almost none.

Apoptosis: The amount of apoptosis determined as the sub-G1 fraction by DNA flow cytometry at $24 \mathrm{~h}$ comprises $7 \%$ for WI-L2-NS and $15 \%$ for TK6. However later the difference increases further: at $72 \mathrm{~h} 65 \%$ of TK6 cells are in apoptosis, while only $25 \%$ of WI-L2-NS cells are apoptotic.

Topological analysis of the DNA repair by HR: Repair foci were observed in both cell-lines within 5 min postdamage. Notably, they were first assembled from diffuse forms of g-H2AX and Rad51 at the interface between the condensed chromatin and the interchromatin (nuclear matrix) space. Larger areas of g-H2AX and Rad51 were observed in WI-L2-NS 5 min after damage and the average number of repair foci per cell was 2.2 as compared with 1.0 for TK6 cells (Table 1).

Table 1. Kinetic analysis of g-H2AX and Rad51 components after irradiation determined by immunofluorescent staining and image analysis. Only foci containing both $\mathrm{g}-\mathrm{H} 2 \mathrm{AX}$ and Rad51 components were scored as repair foci.

\begin{tabular}{|c|c|c|c|c|c|c|c|c|}
\hline $\begin{array}{c}\text { Time after } \\
\text { irradiation }\end{array}$ & \multicolumn{2}{|r|}{5 min } & \multicolumn{2}{|c|}{6 h } & \multicolumn{2}{r|}{$24 \mathrm{~h}$} & \multicolumn{2}{r|}{$72 \mathrm{~h}$} \\
\hline Cell line & TK6 & $\begin{array}{l}\text { WI- } \\
\text { L2- } \\
\text { NS }\end{array}$ & TK6 & $\begin{array}{l}\text { WI- } \\
\text { L2- } \\
\text { NS }\end{array}$ & TK6 & $\begin{array}{r}\text { WI- } \\
\text { L2- } \\
\text { NS }\end{array}$ & TK6 & $\begin{array}{l}\text { WI- } \\
\text { L2- } \\
\text { NS }\end{array}$ \\
\hline $\begin{array}{c}\text { Number of } \\
\text { repair } \\
\text { foci/cell }\end{array}$ & 1.0 & 2.2 & 1.5 & 3.2 & 8.8 & 8.8 & 3.6 & 6.1 \\
\hline $\begin{array}{c}\text { Average area } \\
\text { of Rad51 foci } \\
\text { (pxl) }\end{array}$ & 38.4 & 45.1 & 51.0 & 56.8 & 52 & 53.6 & 43.9 & 49.3 \\
\hline $\begin{array}{c}\text { Area } \\
\text { occupied by } \\
\text { Rad51/ cell }\end{array}$ & 37.6 & 98.8 & 78.9 & 182.7 & 456 & 471 & 160 & 301 \\
\hline $\begin{array}{c}\text { Area } \\
\text { occupied by } \\
\gamma-\mathrm{H} 2 A X / c e l l\end{array}$ & 14.0 & 54.6 & 584 & 319.0 & 663 & 513 & 196 & 328 \\
\hline
\end{tabular}

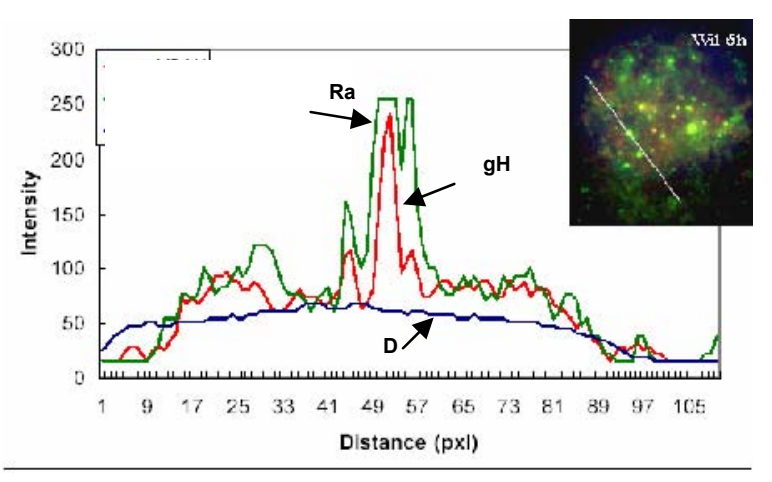

Fig.1. Segmentation of g-H2AX repair foci saturated with Rad51 in a WI-L2-NS cell, $6 \mathrm{~h}$ post-irradiation

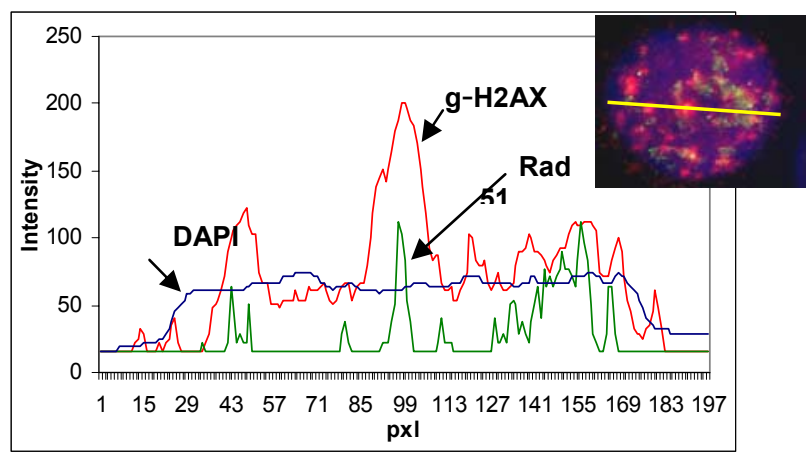

Fig.2. Segmentation through g-H2AX foci binding negligible amounts of Rad51 in a TK6 cell, 6 h post-irradiation

More effective formation of repair foci in WI-L2-NS was also observed at $6 \mathrm{~h}$ post-damage ( 3.2 versus 1.5 per nucleus) and notably, at this term the sum area occupied by $\mathrm{g}-\mathrm{H} 2 \mathrm{AX}$ in TK6 was seven-fold higher than the sum area of the Rad51 label, while this difference in WI-L2NS cells was only 1.7 (Table 1 ).

The difference was also seen by one-line, three-colour (red, green, blue) scanning through the repair foci. In WIL2-NS the highly colocalized concentration of g$\mathrm{H} 2 \mathrm{AX} / \mathrm{Rad} 51$ sharply defined the functional repair foci (Fig.1).

In contrast, Rad51 was much less prevalent in the centre of large $\mathrm{g}-\mathrm{H} 2 \mathrm{AX}$ foci in many irradiated TK6 cells (Fig.2).

The g-H2AX-labelled foci, which were free of Rad51, tended to cluster and fuse forming the chromatin pattern typical of early apoptotic cells (Fig.3).

The discrepancy between the cell lines was diminished at $24 \mathrm{~h}$, when similar amounts of cells were arrested in the G2 cell cycle compartment. At this time, both cell-lines showed similar levels of g-H2AX and Rad51 staining and the highest average number of repair foci per nucleus (8.8) 


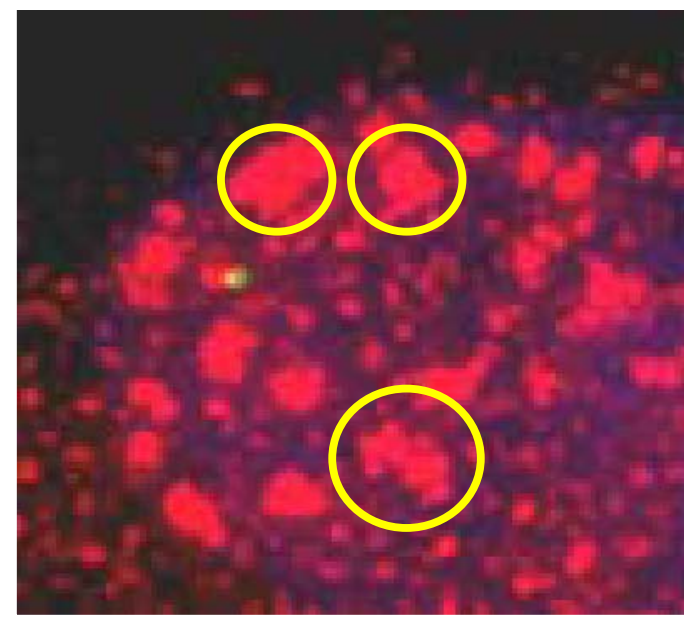

Fig.3. Clustering and fusion of multiple g-H2AX foci in a pre-apoptotic TK6 cell nucleus.

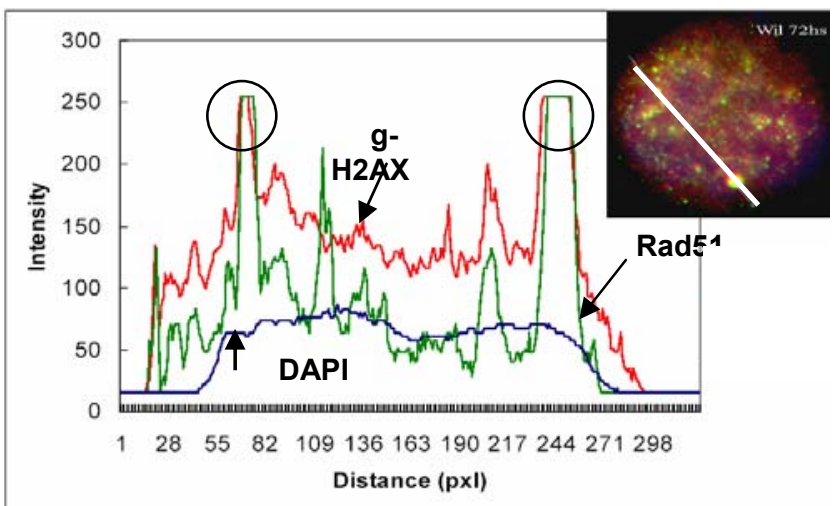

Fig.4. Giant repair foci segmented in a polyploid WI-L2-NS cell nucleus, 72 $\mathrm{h}$ post irradiation.

was apparent (Table 1). Some cell nuclei in both cell lines became free of both components (presumably having repaired the DSB). However, at $72 \mathrm{~h}$, the difference in HR foci again showed the higher repair potential of the WI-L2-NS cells (6.1 versus 3.6 foci in TK 6 cells). The average size of Rad51 foci in TK6 cells between 6-72 $\mathrm{h}$ was determined to be statistically smaller by the non-parametrical sign test $(p<0.001)$. At $72 \mathrm{~h}$, some of WI-L2-NS polyploid cells displayed giant repair foci of heterogenous size, with high concentration of the both components and increased local DAPI binding (Fig.4).

\section{DiscusSION}

Here we analysed the DNA damage response in two different lymphoblastoid cell lines derived from the same source which differ in their p53 status. After ionising irradiation, both cell lines formed HR repair foci containing colocalized g-H2AX and Rad51. However, the p53 competent TK 6 cells had a reduced ability to target Rad51 to g-H2AX-signaling foci coincident with the timing of the first (6h) and further (72h) S phases. Recombination in S-phase is assumed to be illegitimate as it favours mutation. Mutations arising as a result of enhanced recombination repair were found previously in WI-L2-NS cells as compared with TK6 [4]. More recently, a specific helicase Srs2 has been discovered which normally prevents uploading of $\operatorname{Rad} 51$ to the DNA replication processivity clamp [5]. Our data indirectly suggest that this mechanism may be p53-dependent, while phosphorylation of $\mathrm{H} 2 \mathrm{AX}$ in the damage-signalling cascade may be independent of p53. The topological consequence of the restricted association of Rad51 with the g-H2AX foci was evidenced by their structural deterioration, multiplication of $\mathrm{g}-\mathrm{H} 2 \mathrm{AX}$ domains and decrease in DAPI binding. In contrast, the unrestricted binding of Rad51 to g-H2AX repair sites in p53 mut polyploid WI-L2-NS cells leads to the formation of enormous, well-shaped foci and notably - to the local increase of DAPI binding. DAPI is a DNA intercalating dye whose binding is dependent on both its DNA content and double-strandedness: DAPI does not bind singlestranded DNA. Therefore, our observations suggest that Rad51 may prevent relaxation of the DNA in the HR repair foci. Possibly, Rad51 filaments may penetrate into the sites of damage, locating single strand breaks (SSB) searching for the homology to 'plug' the breaks, thus favouring staining with DAPI. The relaxation of chromatin in the Rad51-depleted g-H2AX-foci was observed by us as the initiating step switching the chromatin from attempts of HR towards the initiation of apoptosis in the p53-competent TK6 cells. Excessive g$\mathrm{H} 2 \mathrm{AX}$ formation was shown to appear during apoptosis concurrently with the initial appearance of high molecular weight DNA fragments [6]. These high molecular weight DNA fragments observed during apoptosis are produced by topoisomerase II (Topo II). Paradoxically, Topo II has also recently been linked with preventing mutagenic DNA repair by illegitimate non-homologous recombination [7]. Therefore, currently the temporal regulation of this process is unclear - i.e. whether initial apoptosis started by Topo II is then interrupted by binding of Rad51 to SSB (shifting Topo II activity from DNA cleavage to religation) or the whether the DNA repair by HR fails, releasing $\operatorname{Rad} 51$ and thus allowing apoptosis to initiate (via Topo II). Regardless, it seems clear that protection from apoptosis by HR in p53-deficient tumor cells occurs in the chromatin providing these cells with genotoxic resistance and increased mutagenicity, thus promoting tumor progression. 


\section{CONCLUSION}

A restriction in binding of Rad51 recombinase to g-H2AX HR repair foci was observed in S-phase in p53 wt TK6 lymphoma cells, compared to the p53 mutated counterpart WI-L2-NS. The inability to stably bind Rad51 leads to a visible relaxation of the DNA resulting in the multiplication and fusion of g-H2AX domains in early apoptotic chromatin. These data indicate that prevention of apoptosis in $\mathrm{p} 53$ deficient tumor cells occurs through HR at the level of the chromatin.

\section{AcKNOwledgment}

Grant 05.1634 and 05.1634 .1 of the Latvian Scientific Council supported this research in Riga, the exchange visit grant of the Royal Society of London and Cancer Research Foundation, in Southampton and Manchester, UK.

\section{REFERENCES}

1. Raderschall E, Bazarov A, Cao J et al (2002) Formation of higher-order nuclear Rad51 structures is functionally linked to p21 expression and protection from DNA damage-induced apoptosis. J Cell Sci 115:153164
2. Ivanov A, Cragg MS, Erenpreisa J et al (2003) Endopolyploid cells produced after severe genotoxic damage have the potential to repair DNA double strand breaks. J Cell Sci 116: 4095-4106

3. Paull T, Rogakou EP, Yamazaki V, Kirchgessner CU et al (2000) A critical role for histone $\mathrm{H} 2 \mathrm{AX}$ in recruitment of repair factors to nuclear foci after DNA damage Curr Biol 10:886-895

4. Xia F, Amundson SA, Nickoloff JA et al (1994) Different capacities for recombination in closely related human lymphoblastoid cell lines with different mutational responses to X-irradiation. Mol Cell Biol 14:5850-5857

5. Pfander B, Moldovan GL, Sacher M et al (2005) SUMO-modified PCNA recruits Srs2 to prevent recombination during $\mathrm{S}$ phase. Nature 436:428-433

6. Rogakou EP, Nieves-Neira W, Boon C et al (2000) Initiation of DNA fragmentation during apoptosis induces phosphorylation of H2AX histone at serine 139. J Biol Chem 275:9390-9395

7. Umanskaya ON, Lebedeva SS, Gavrilov AA et al (2006) Inhibition of DNA topoisomerase II may trigger illegitimate recombination in living cells: experiments with a model system. J Cell Biochem 99:598-608

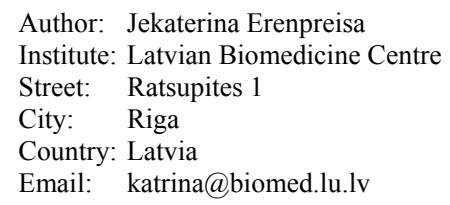

